\title{
A Review of Driver Mental Workload in Driver-Vehicle-Environment System
}

\author{
Yutao Ba and Wei Zhang \\ Department of Industrial Engineering, Tsinghua University, Beijing, R.P. China \\ bb0438@163.com, zhangwei1968@gmail.com
}

\begin{abstract}
This paper reviews mental workload literatures which focus on the field of driving task. The purpose is to identify and discuss shortcomings in present research and opportunities for future study. A comprehensive search of journal papers on ISI Web of Science ${ }^{\circledR}$ (WoS) data base was conducted. 50 most-cited studies [1-50] are analyzed and classified into 4 categories: characteristics of the driver, characteristics of the vehicle, characteristics of environment, measurement and modeling. Chronological historiograph is generated to highlight the relationship among these studies in the retrieved categories. We argue that the ambiguity of concept and dissociative measurement are still primary barriers for current workload research. The further application of driver mental workload research should fully consider various potential influence factors in the Driver-Vehicle-Environment (DVE) system.
\end{abstract}

\section{Introduction}

Driving behavior is a direct consequence of the stimuli received from the road infrastructure, surrounding environment and atmosphere inside the vehicle [51]. Currently, the evaluation of mental workload is a key point among the researches to the development of the Driver-Vehicle-Environment (DVE) System. The intent of this paper, therefore, is to provide a review of the mental workload literatures, which focus on the field of driving task.

The most organized discussion of mental workload can be found in the "Handbook of Perception and Human Performance" [52,54]. De Waard [51] conducted detailed overview of general characteristics of driver mental workload measures. In previous reviews, there is often a tendency to be highly technology driven and to focus primarily on methodology and measurement, while reflecting less on inherent relevance of current interrelated researches. Inspired by related studies within the field of road safety, we aim at evoking more discussion about effects of driver, vehicle, and environment characteristics on mental workload by presenting a snapshot, identifying and discussing shortcomings in the present research and opportunities for the future study. This paper is organized as follow. Section 2 begins with concept and fundamental researches about mental workload. Three aspects of mental workload are briefly viewed. In section 3, we conduct a comprehensive search of journal papers. 50 most-cited studies [1-50] are analyzed and classified into 4 
categories. Chronological historiograph is generated to highlight the relationship among these studies in the retrieved categories. Characteristics of the driver, characteristics of the vehicle, characteristics of environment, measurement and predictive model are then discussed in section 4, 5 and 6 . Section 7 concludes with discussion of limitations in the reviewed research and potential trend in the future study.

\section{Fundamental Researches of Mental Workload}

Since the 1970s, the interest in defining and developing measures of mental workload has increased dramatically. Nevertheless, with about 40 years' interest in workload research, there is still no clearly-defined, universally-accepted definition of mental workload. Roscoe [55] provided an earlier review which acknowledges task demands, operator capabilities, and contextual temporal demands as being components of workload. Gopher and Braune [53] suggested that the workload construct is conceived to explain the inability of human operators to cope with the requirements of a task, and that workload measurement is an attempt to characterize performance of the task relative to the operator's capability. For those reason, three aspects of mental workload should be considered.

1. Task demand: tasks complexity for limited human capacity.

2. Performance: primary-task or secondary-task performance.

3. Effort: the strategy and conscious allocation of mental processing resources.

Workload measurement techniques are typically organized into three broad categories: self-assessment, performance measures and physiological measures. It has already been noted that different measures are sensitive to different aspects of workload and not all workload measures are assessing the same thing. In De Warrd's [51] review, different mental workload measures were presented and evaluated on their potential use as indicator of workload in traffic research.

\section{Method}

In this paper, a comprehensive search of journal papers on ISI Web of Science ${ }^{\circledR}$ (WoS) data base is conducted. To be included in this collection, a study has to (1) aim at studying mental workload of road driving task and (2) have great impact on subsequent researches, which is measured by Global Citation Score (GCS), the total number of citations to a paper in the WoS.

While operating a driving task, the information can come from the driver itself, current interactions, traffic environment and road scenario. As the result, the drivers' metal workload is influenced by the characteristics of driver, vehicle and surrounding environment. In addition, some studies consider those characteristics as a whole and focuse on developing a general measurement and constructing a predictive model of driver's mental workload. According to this thought, the topics of reviewed studies were categorized into 4 domains: characteristics of the driver, characteristics of the vehicle, characteristics of environment and measurement and modeling. Some of 


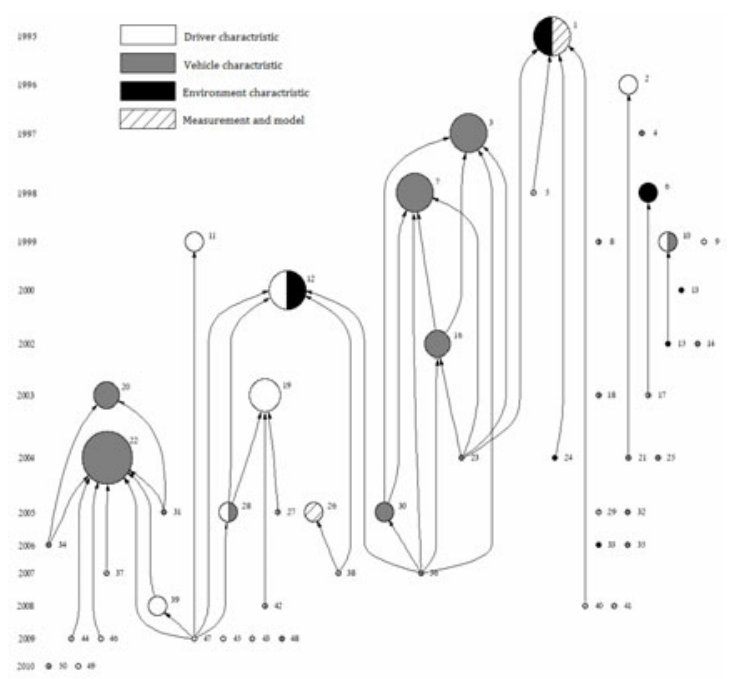

Fig. 1. Chronological historiographs of 50 most-cited reviewed literatures

reviewed studies belong to more than one category. A total of 50 most-cited studies [1-50] were reviewed and analyzed in this article. WoS export files were created in which all cited references for each source document were captured. We generated a chronological historiographs (Fig. 1) for those most-cited studies to highlight the relationship between these researches in the retrieved category.

\section{Characteristics of Driver}

Visual and information processing are the most important driver capacities for safe driving. Several researchers aimed at constructing a clear relationship between these capacities with mental workload. Liu [1] pointed out that the effects of visual scanning on workload are more pronounced in the dual task conditions than in the single one. In addition, Increases in scanning demand produce greater interference to a concurrent spatial task than to a verbal task. Recarte and Nunes [20] pushed this research to a step forward. They studied the effects of mental workload on visual search, discrimination and decision making in real traffic conditions. Results indicated that the increased workload produce endogenous distraction and spatial gaze concentration, affecting the capacity to process visual stimuli.

Age is associated with declines in perceptual, response time, cognitive memory, attention, physical strength and dexterity performance. Hakamies-Blomqvist, et al. [9] used instrumented car to test the age related differences in organizing car-controlling movements. The experiment result showed that older drivers organize their carcontrolling movements in a more serial way in order to reduce the momentary mental workload produced by complex traffic situations. Makishita and Matsunaga [40] examined differences in reaction time of various age groups to assess the influence of 
mental workload on reaction time. Results suggested that high mental workload increase the average reaction time for each age group, whereas mental workload influences elderly drivers' reaction time remarkably.

Other aspects of driver characteristics were also considered, such as injury experience, driving skills and training. Riese, et al. [10] focused on patients with very severe Closed Head Injury (CHI). Young and Stanton [16] analyzed how the development of automaticity within the driving task may influence performance of different levels of driver skill and workload. Wang, et al. [50] found that subjective mental workload of the trained drivers was significantly lower than novice driver in completing the simulated driving task of road hazard handling.

\section{Characteristics of Vehicle}

Nowadays, great challenge of increased demands during driving task is caused by the introduction of information system into the vehicle. All these In-vehicle Information System (IVIS) require drivers' attention to be divided between the system and the primary task of vehicle control. In an experiment of Lansdown, et al. [21], participants were presented with a primary task, representing some of the visual and manual aspects of driving, and three secondary tasks (visual, auditory, and visual \& auditory stimuli), representing the aspects of in-vehicle system operation. Differences between the impacts of the three secondary tasks were not observed. Addition research [26] proved that analogous auditory and visual secondary tasks were of equivalent difficulty. Koo, et al. [45] studied the effect of voice or display information systems on drivers through the driver eye movement. Result suggested that effect of high workload produced by telematics services focuses on driver's object visual field. The effects of different display modes were compared between Head-Up Display (HUD) and Head-Down Display (HDD) [22]. Results indicated that using the HUD caused less workload for the drivers than the HDD.

Mobile telephones are most tangible devices that can distract the driver and change the workload. The effect of three types of cell phones (hand held, hands free with an external speaker and personal hands free) on total subjective workload and intelligibility was measured using the NASA-Task Load Index (TLX) and the modified rhyme test (MRT) [19]. In this experiment, the drivers rated all components of workload for each type of cell phone to be significantly higher than for a control condition in which no cell phone was used. It was also concluded that hands free cell phone would interfere least with the cognitive demands of driving. Patten, et al. [23] pointed out that the content of the conversation was far more important for driver distraction than the type of telephone. The more difficult and complex the conversation, the greater the possible negative effect on increment in workload. Tornros and Bolling [32] found driving speed decreased as an effect of dialing with the greatest effect for hands free phone mode. They interpreted effects on speed as a compensatory effort for the increased mental workload.

Vehicle automation is another irresistible trend in this century with undoubted benefits. The early study of the vehicle automation effect on mental workload was since 1990s. Stanton and Young [6] presented a review of vehicle automation. They shown a reduction in mental workload associated with some forms of automation. 
Since then, much effort was spent on specific automation device. Ma and Kaber [30] verified the effects of an adaptive Cruise Control (ACC) system on situation awareness and mental workload. Their results was different from Stanton and Yong [31], which indicated use of the ACC system improves driving task situation awareness (SA) under typical driving conditions, and reduces driver mental workload. Young and Stanton [38] exposited the adaptive nature of the ACC system to reconcile this contradiction and advised to use variable-speed tasks to ensure that all aspects of device functionality are covered. The effect of automated system of bus docking on drivers' mental workload was also tested [18]. Results showed that docking precision is improved when the system is used. When drivers monitored the functioning of the system, their workload was higher than that observed during manual docking. However, reduced workload was evidenced after a learning process. It's interesting that the docking system was shown to increase workload highly in the event of dysfunction, especially when drivers had to take over control. A similar experiment [35] was also conducted on Lane Support System (LSS). The general suggestions about automation design in vehicle were given by Young and Stanton [16]: according to proposed malleable attention resources theory, future vehicle designers should employ their technology in driver support systems rather than in automation to replace the driver.

\section{Characteristics of Environment}

Verwey [13] suggested that road situation is a major determinant of visual and mental workload of the driver. Numerous specific road characteristics were studied separately, including road curvature change rate, road mark, roadside advertising etc. Richter, et al. [5] and Backs, et al. [17] introduced the road curvature change rate as an independent variable, which served as a criterion of objective road difficulty. Both psycho-physiological variables (heart rate, blink rate, skin conductance response) and speed varied as a function of the curvature change rate of the road segments, indicating that driving performance significantly deteriorated and visual demand significantly increased as curve radius decreased. Steyvers and De Waaed [12] compared two types of road-edge delineation, continuous or dashed edge lines with two control roads without lines or with only a dashed line on the road axis. Subjectively rated effort was higher for the unlined control road than for the three other roads. It was concluded that edge-lines may provide a simple and effective way of inducing a more favorable lateral position on rural roads without having negative effects on mental workload. Another comparison of an enhanced road-marking system with commonly used highway markings was undertaken by Horberry, et al. [33]. A similar pattern was found for the subjective measures: workload was rated lower for the enhanced markings.

Some specific driving scenarios were also considered. Horrey and Simons [36] examined the impact of mental workload on safety margins (distances) that drivers keep when engaged in a tactical control task (passing other vehicles). The result show although drivers did increase their headway adaptively when engaged in steady-state car following, they did not adapt their behavior to accommodate mental workload when performing tactical control maneuvers and implicate the difference between 
steady-state and tactical control driving contexts. Some studies [39] focused on assessing effects of Heavy Goods Vehicles (HGVs) on other drivers' behavior and mental workload during filtering into traffic and exiting from the motorway. It was concluded that an increase in HGVs will make merging into and exiting from traffic more mentally demanding and will decrease safety margins.

\section{Measurement and Modeling}

The systemic overview of drivers' workload measurement can be found in De waard 's [51] handbook "The Measurement of Drivers Mental Workload" on the basis of the following properties: sensitivity, diagnosticity, primary-task intrusion, implementation requirements and operator acceptance. Later studies were almost following this framework. In Backs' study [17], cardiac, performance, and visual demand measures of driver workload were obtained. The patterns of dissociation were interpreted as being capable of isolating the perceptual demands of driving from the central and motor processing demands. Several studies $[23,28]$ using the Peripheral Detection Task (PDT) method to measure driver workload compare with subjective workload ratings as well as physiological measures. Result suggested that the PDT proved to be sensitive to peaks in workload; subjective workload ratings reflected overall route demands; physiological measures were less sensitive to workload and indicated emotional strain as well. Di Stasi et al. [49] found that saccadic peak velocity could be a useful diagnostic index for the assessment of operators' mental workload and attention state in hazardous environments. Mehler, et al. [46] examined the sensitivity of heart rate, skin conductance, and respiration rate as measures of mental workload in a simulated driving environment. The pattern of results indicated that physiological measures can be sensitive to changes in workload before the appearance of clear decrements in driving performance. On the whole, consensus of current investigations indicates that a battery of assessment techniques will provide the most sensitive assessment of workload in complex environments. Meanwhile, with the technical development in the area of physiology, more and more physiological measures are recommended.

Several conceptual and numerical models of mental workload have been introduced sine 1990s. Zeitlin [1] conducted a long-term field trial to estimates driver mental workload. Increment of mental workload was a function of traffic density, average speed, and uncertainty (estimated by the number of brake depressions). Following this result, he [7] suggested that driving workload has two components, a steady-state load dictated by road conditions, speed, and traffic density and a transient load determined by the degree of uncertainty in the driving situation. An objective workload index of the general form, workload $=\mathrm{F}$ (brake actuation rate $+\log (2)$ speed), based on this model of driver behavior predicts subjective driving difficulty and workload. The concept of driving task difficulty [27] is elaborated within the framework of the Task-Capability Interface (TCI) model. In this model, task difficulty homeostasis was proposed as a key sub-goal in driving and speed choice was argued to be the primary solution to the problem of keeping workload within selected boundaries. Several more sophisticated numerical models, including artificial neural network architectures [29] and queuing network [37] were used to simulate drivers' mental workload and the performance. However, the accuracy of these numerical models still needs to be improved. 


\section{Discussion and Conclusion}

With about 40 years development, the concept that mental workload is multidimensional is not seriously challenged today. Meanwhile, there still have not been any major developments in the understanding or measurement of mental workload in the recent literatures. The recent techniques used to measure mental workload are similar to those used over the past 30 years, namely subjective measures, performance measures and physiological measures. When measuring workload empirically, the current recommendations are largely the same as twenty years ago: a battery of assessment techniques will provide the most sensitive assessment of workload in complex environments [52]. However, with the technical development in the area of biomedicine and physiology, more and more psychophysiological measures are recommended for application. It's necessary to develop a formal, unified theory that can explain the interactions of various physiological phenomena and their relationship to workload.

On the other hand, drivers' metal workload is influenced by characteristics of the driver, vehicle and surrounding environment in the DVE system. As to characteristics of the driver, studies in this domain concentrated upon constructing a clear relationship of driver capacities with mental workload, considering individual difference such as age, skill and experience as influence factors.

Meanwhile, increased demands are from the introduction of new technology into the vehicle, primarily including IVIS and vehicle automation. Information form (visual, auditory, and visual \& auditory) and display modes are hotspots of the IVIS researches. Although vehicle automation is suggested by most investigators to be able to improve performance and reduce mental workload, overburden workload may occur in the dysfunction of automation, especially when drivers had to take over control. In general, further investigation of these human-vehicle interactions can offer fundamental guidance for efficiency and safety vehicle design without negative effects on mental workload.

Looking at studies concerning with traffic environment, assessment of driving task demands and mental workload in different traffic condition is consider as the starting point for road design. A lot of specific road characteristics were studies separately, including road curvature change rate, road mark, roadside advertising, etc. Some specific driving scenarios (steady-state car following and tactical control maneuvers) are also considered. However, there are still enormous opportunities for future study in road situation and road construction.

In conclusion, this review examines mental workload literatures which focus on the field of driving task. Large numbers of impact factors among the DVE system on driver's mental workload have been considered in recent studies. But there have not been any conceptual model to interpret the inner link between those characteristics. An important reason contributing to this situation is fuzzy concept of mental workload. To measure, assess and predict workload accurately, the further research of mental workload research should fully considering the impact factor from driver, vehicle device, road environment and scenario as an uniform input of human information process. 


\section{References}

1. Zeitlin, L.R.: Estimates of Driver Mental Workload: A Long-Term Field Trial of Two Subsidiary Tasks. Human Factors 37(3), 611-621 (1995)

2. Liu, Y.L.: Quantitative Assessment of Effects of Visual Scanning on Concurrent Task Performance. Ergonomics 39(3), 382-399 (1996)

3. Cha, D., Park, P.: User Required Information Modality and Structure of In-Vehicle Navigation System Focused on The Urban Commuter. Computers \& Industrial Engineering 33(3-4), 517-520 (1997)

4. Stanton, N.A., Young, M., McCaulder, B.: Drive-by-Wire: The Case of Driver Workload and Reclaiming Control with Adaptive Cruise Control. Safety Science 27(2-3), 149-159 (1997)

5. Richter, P., Wagner, T., Heger, R., Weise, G.: Psychophysiological Analysis of Mental Load during Driving on Rural Roads - a Auasi-Experimental Field Study. Ergonomics 41(5), 593-609 (1998)

6. Stanton, N.A., Young, M.S.: Vehicle Automation and Driving Performance. Ergonomics 41(7), 1014-1028 (1998)

7. Zeitlin, L.R.: Micromodel for Objective Estimation of Driver Mental Workload from Task Data. In: Driver and Vehicle Modeling, pp. 28-34 (1998)

8. De Waard, D., Van der Hulst, M., Brookhuis, K.A.: Elderly and Young Drivers' Reaction to an In-car Enforcement and Tutoring System. Applied Ergonomics 30(2), 147-157 (1999)

9. Hakamies-Blomqvist, L., Mynttinen, S., Backman, M., Mikkonen, V.: Age-Related Differences in Driving: Are Older Drivers more Serial? International Journal of Behavioral Development 23(3), 575-589 (1999)

10. Riese, H., Hoedemaeker, M., Brouwer, W.H., Mulder, L.J.M., Cremer, R., Veldman, J.B.P.: Mental Fatigue after Very Severe Closed Head Injury: Sustained Performance, Mental Effort, and Distress at Two Levels of Workload in a Driving Simulator. Neuropsychological Rehabilitation 9(2), 189-205 (1999)

11. Verwey, W.B., Zaidel, D.M.: Preventing Drowsiness Accidents by an Alertness Maintenance Device. Accident Analysis and Prevention 31(3), 199-211 (1999)

12. Steyvers, F., de Waard, D.: Road-edge Delineation in Rural Areas: Effects on Driving Behaviour. Ergonomics 43(2), 223-238 (2000)

13. Verwey, W.B.: On-line Driver Workload Estimation. Effects of Road Situation and Age on Secondary Task Measures. Ergonomics 43(2), 187-209 (2000)

14. Lansdown, T.C., Brook-Carter, N., Kersloot, T.: Primary Task Disruption from Multiple In-vehicle Systems. Its Journal 7(2), 151-168 (2002)

15. Tejero, P., Choliz, M.: Driving on the Motorway: the Effect of Alternating Speed on Driver's Activation Level and Mental Effort. Ergonomics 45(9), 605-618 (2002)

16. Young, M.S., Stanton, N.A.: Malleable Attentional Resources Theory: A new Explanation for the Effects of Mental Underload on Performance. Human Factors 44(3), 365-375 (2002)

17. Backs, R.W., Lenneman, J.K., Wetzel, J.M., Green, P.: Cardiac Measures of Driver Workload During Simulated Driving with and without Visual Occlusion. Human Factors 45(4), 525-538 (2003)

18. Collet, C., Petit, C., Champely, S., Dittmar, A.: Assessing Workload Through Physiological Measurements in Bus Drivers Using an Automated System During Docking. Human Factors 45(4), 539-548 (2003) 
19. Matthews, R., Legg, S., Charlton, S.: The Effect of Cell Phone Type on Drivers' Subjective Workload during Concurrent Driving and Conversing. Accident Analysis and Prevention 35(4), 451-457 (2003)

20. Recarte, M.A., Nunes, L.M.: Mental Workload while Driving: Effects on Visual Search, Discrimination, and Decision Making. Journal of Experimental Psychology-Applied 9(2), 119-137 (2003)

21. Lansdown, T., Brook-Carter, N., Kersloot, T.: Distraction from Multiple In-vehicle Secondary Tasks: Vehicle Performance and Mental Workload Implications. Ergonomics 47(1), 91-104 (2004)

22. Liu, Y.C., Wen, M.H.: Comparison of Head-up Display (HUD) vs. Head-Down Display (HDD): Driving Performance of Commercial Vehicle Operators in Taiwan. International Journal of Human-Computer Studies 61(5), 679-697 (2004)

23. Patten, C.J.D., Kircher, A., Ostlund, J., Nilsson, L.: Using Mobile Telephones: Cognitive Workload and Attention Resource Allocation. Accident Analysis and Prevention 36(3), 341-350 (2004)

24. Paul, P., Kuijer, F.M., de Vries, W.H.K., van der Beek, A.J., van Dieen, J.H., Visser, B., Frings-Dresen, M.H.W.: Effect of Job Rotation on Work Demands, Workload, and Recovery of Refuse Truck Drivers and Collectors. Human Factors 46(3), 437-448 (2004)

25. Young, M., Stanton, N.: Taking the Load off: Investigations of How Adaptive Cruise Control Affects Mental Workload. Ergonomics 47(9), 1014-1035 (2004)

26. Baldwin, C.L., Coyne, J.T.: Dissociable Aspects of Mental Workload: Examinations of the P300 ERP Component and Performance Assessments. Psychologia 48(2), 102-119 (2005)

27. Fuller, R.: Towards a General Theory of Driver Behaviour. Accident Analysis and Prevention 37(3), 461-472 (2005)

28. Jahn, G., Oehme, A., Krems, J.F., Gelau, C.: Peripheral Detection as a Workload Measure in Driving: Effects of Traffic Complexity and Route Guidance System Use in a Driving Study. Transportation Research Part F-Traffic Psychology and Behaviour 8(3), 255-275 (2005)

29. Lin, Y., Tang, P., Zhang, W.J., Yu, Q.: Artificial Neural Network Modelling of Driver Handling Behaviour in a Driver-Vehicle-Environment System. International Journal of Vehicle Design 37(1), 24-45 (2005)

30. Ma, R.Q., Kaber, D.B.: Situation Awareness and Workload in Driving while Using Adaptive Cruise Control and a Cell Phone. International Journal of Industrial Ergonomics 35(10), 939-953 (2005)

31. Stanton, N.A., Young, M.S.: Driver Behaviour with Adaptive Cruise Control. Ergonomics 48(10), 1294-1313 (2005)

32. Tornros, J.E.B., Bolling, A.K.: Mobile Phone Use - Effects of Handheld and Hands Free Phones on Driving Performance. Accident Analysis and Prevention 37(5), 902-909 (2005)

33. Horberry, T., Anderson, J., Regan, M.A.: The Possible Safety Benefits of Enhanced Road Markings: A Driving Simulator Evaluation. Transportation Research Part F-Traffic Psychology and Behaviour 9(1), 77-87 (2006)

34. Tornros, J., Bolling, A.: Mobile Phone Use - Effects of Conversation on Mental Workload and Driving Speed in Rural and Urban Environments. Transportation Research Part FTraffic Psychology and Behaviour 9(4), 298-306 (2006)

35. Ward, N.J., Shankwitz, C., Gorgestani, A., Donath, M., De Waard, D., Boer, E.R.: An Evaluation of a Lane Support System for Bus Rapid Transit on Narrow Shoulders and The Relation to Bus Driver Mental Workload. Ergonomics 49(9), 832-859 (2006)

36. Horrey, W.J., Simons, D.J.: Examining Cognitive Interference and Adaptive Safety Behaviours in Tactical Vehicle Control. Ergonomics 50(8), 1340-1350 (2007) 
37. Wu, C.X., Liu, Y.L.: Queuing Network Modeling of Driver Workload and Performance. IEEE Transactions on Intelligent Transportation Systems 8(3), 528-537 (2007)

38. Young, M.S., Stanton, N.A.: What's Skill Got to Do with It? Vehicle Automation and Driver Mental Workload. Ergonomics 50(8), 1324-1339 (2007)

39. De Waard, D., Kruizinga, A., Brookhuis, K.A.: The Consequences of an Increase in Heavy Goods Vehicles for Passenger Car Drivers' Mental Workload and behaviour: A Simulator Study. Accident Analysis and Prevention 40(2), 818-828 (2008)

40. Makishita, H., Matsunaga, K.: Differences of Drivers' Reaction Times According to Age and Mental workload. Accident Analysis and Prevention 40(2), 567-575 (2008)

41. Recarte, M.A., Perez, E., Conchillo, A., Nunes, L.M.: Mental Workload and Visual Impairment: Differences between pupil, blink, and subjective rating. Spanish Journal of Psychology 11(2), 374-385 (2008)

42. Wickens, C.D.: Multiple resources and Mental Workload. Human Factors 50(3), 449-455 (2008)

43. Cantin, V., Lavalliere, M., Simoneau, M., Teasdale, N.: Mental Workload when Driving in a Simulator: Effects of Age and Driving Complexity. Accident Analysis and Prevention 41(4), 763-771 (2009)

44. Horrey, W.J., Lesch, M.F., Garabet, A.: Dissociation between Driving Performance and Drivers' Subjective Estimates of Performance and Workload in Dual-task Conditions. Journal of Safety Research 40(1), 7-12 (2009)

45. Koo, T.Y., Park, K.J., Kim, B.Y., Kim, H.J., Suh, M.W.: A Study on Driver's Workload of Telematics Using a Driving Simulator: A Comparison among Information Modalities. International Journal of Precision Engineering and Manufacturing 10(3), 59-63 (2009)

46. Mehler, B., Reimer, B., Coughlin, J.F., Dusek, J.A.: Impact of Incremental Increases in Cognitive Workload on Physiological Arousal and Performance in Young Adult Drivers. Transportation Research Record (2138), 6-12 (2009)

47. Pellegrino, O.: An Analysis of the Effect of Roadway Design on Driver's Workload. Baltic Journal of Road and Bridge Engineering 4(2), 45-53 (2009)

48. Reimer, B.: Impact of Cognitive Task Complexity on Drivers' Visual Tunneling. Transportation Research Record (2138), 13-19 (2009)

49. Di Stasi, L.L., Renner, R., Staehr, P., Helmert, J.R., Velichkovsky, B.M., Canas, J.J., Catena, A., Pannasch, S.: Saccadic Peak Velocity Sensitivity to Variations in Mental Workload. Aviation Space and Environmental Medicine 81(4), 413-417 (2010)

50. Wang, Y.B., Zhang, W., Salvendy, G.: Effects of a Simulation-based Training Intervention on Novice Drivers' Hazard Handling Performance. Traffic Injury Prevention 11(1), 16-24 (2010)

51. De Waard, D.: The Mmeasurement of Drivers' Mental Workload. Ph.D.thesis. University of Groningen, Traffic Research Centre, Haren, Netherlands (1996)

52. Gopher, D., Donchin, E.: Workload -An Examination of the Concept. In: Boff, K.R., Kaufman, L., Thomas, J.P. (eds.) Handbook of Perception and Human Performance. Cognitive Processes and Performance, vol. II. Wiley, New York (1986)

53. Gopher, D., Braune, R.: On the Psychophysics of Workload-Why Bother with Subjective Measures. Hum. Factors 26(5), 519-532 (1984)

54. O’Donnell, R.D., Eggemeier, F.T.: Workload Assessment Methodology. In: Boff, K.R., Kaufman, L., Thomas, J.P. (eds.) Handbook of Perception and Human Performance. Cognitive Processes and Performance, vol. II. Wiley, New York (1986)

55. Roscoe, A.H.: Assessing Pilot Workload. AGARD (Advisory Group for Aerospace Research and Development), no. 233, ch. 1, NATO (1978) 